\title{
Robust Stability of Neutral System with Mixed Time-Varying Delays and Nonlinear Perturbations Using Delay Decomposition Approach
}

\author{
Fang Qiu and Quanxin Zhang \\ Department of Mathematics, Binzhou University, Shandong 256603, China \\ Correspondence should be addressed to Fang Qiu; rgbayqf@163.com
}

Received 27 January 2014; Revised 8 June 2014; Accepted 18 June 2014; Published 8 July 2014

Academic Editor: Luisa Morgado

Copyright (C) 2014 F. Qiu and Q. Zhang. This is an open access article distributed under the Creative Commons Attribution License, which permits unrestricted use, distribution, and reproduction in any medium, provided the original work is properly cited.

\begin{abstract}
This paper investigates the robust delay-dependent stability problem for neutral system with mixed delays and nonlinear perturbations. A delay decomposition approach is used in this paper in which the information of the delayed plant states can be taken into full consideration. Then, based on a special Lyapunov functional approach, the novel delay-dependent stability criteria are obtained in terms of linear matrix inequalities (LMIs). A numerical example illustrates the effectiveness of the derived method and the improvement over some existing methods.
\end{abstract}

\section{Introduction}

A neutral system that involves time delay in both state and derivatives of state simultaneously is encountered in various areas, including population ecology, heat exchange, and steam processes. Due to its wider application, the problem of the stability of neutral system has received considerable attention by many scholars in recent years [1-12]. Since delaydependent criteria are generally less conservative than delayindependent ones [13], delay-dependent stability analysis for neutral systems has obtained wide attention.

In practice, the systems often contain some uncertainties since it is very difficult to obtain an exact mathematical model due to uncertain, environmental noise or slowly varying parameters, and so forth. Therefore, the robust stability of time-delay systems with nonlinearities has received considerable attention [1-3, 7-11, 14-16]. Various methods aiming at reducing the conservatism of these stability criteria have been proposed. Fixed model transformation was the main method employed in $[1-3,14]$, but these model transformations often introduce additional dynamics which leads to relatively conservative results. The inequality methods were used to estimate the upper bound of cross product terms in the derivative of the Lyapunov functional in $[15,16]$. In order to further improve the performance of stability criteria, free-weighting matrix method was proposed in $\mathrm{He}$ et al. $[4,17,18]$, in which neither system transformation nor bounding technique on some cross terms was involved. However, this method introduced some slack variables apart from matrix variables appearing in Lyapunov-Krasovskii functionals. In addition, the utilization of augmented-type Lyapunov-Krasovskii functionals proposed in $[5,6]$ has provided significant improvements in the stability results for neutral systems, while the above presented method needs to decide more possible variables which will increase the complexity of the computation. When the upper bound of delay derivative may be larger than or equal to 1 , the authors in [19] used a delay decomposition approach and derived new stability results. Motivated by the above discussions, we will consider the stability results of neutral systems for timevarying delays $h(t)$ satisfying $h(t) \in[0, \delta h](\delta<1)$ and $h(t) \in(\delta h, h]$, respectively. Compared with some existing literatures, the delay decomposition method is useful for reducing conservatism of the analysis result.

In this paper, our purpose is to present some new robust delay-dependent stability criteria for neutral systems with mixed delays and nonlinear perturbations. By constructing appropriate Lyapunov-Krasovskii functional based on the delay decomposition approach, some novel delay-dependent stability conditions are derived without resorting to any 
model transformation and free weighting matrix technique. All the stability criteria are expressed in terms of LMIs, which can be solved efficiently by using standard convex optimization algorithms. Finally, a numerical example is given to illustrate the effectiveness and less conservatism of the proposed method.

Notation. Throughout this paper, $T$ stands for matrix transposition. $\mathbf{R}^{n}$ is the $n$-dimensional Euclidean space. $\mathbf{R}^{n \times m}$ is the set of all $n \times m$-dimensional matrices. $I$ denotes the identity matrix of appropriate dimensions. $P>0$ means that $P$ is positive definite. $P \geq 0$ means that $P$ is positive semidefinite. * represents the elements below the main diagonal of a symmetric matrix.

\section{Problem Statement}

Consider the following neutral system with mixed delays and nonlinear perturbations:

$$
\begin{gathered}
\dot{x}(t)=A x(t)+B x(t-h(t))+C \dot{x}(t-d) \\
+f_{1}(x(t), t)+f_{2}(x(t-h(t)), t) \\
+f_{3}(\dot{x}(t-d), t), \\
x(\theta)=\phi(\theta), \quad \dot{x}(\theta)=\varphi(\theta), \\
\forall \theta \in[-\max \{d, h\}, 0],
\end{gathered}
$$

where $x(t) \in \mathbf{R}^{n}$ is the state vector and $A, B, C \in \mathbf{R}^{n \times n}$ are constant matrices with appropriate dimensions. $h(t)$ is a timevarying discrete delay, and it is assumed to satisfy

$$
0 \leq h(t) \leq h, \quad 0 \leq \dot{h}(t) \leq h_{d},
$$

where $h, h_{d}$ are constants. $\phi(\theta), \varphi(\theta)$ are the initial condition functions that are continuously differentiable on $[-\max \{d, h\}, 0] . f_{1}(x(t), t), f_{2}(x(t-h(t)), t)$, and $f_{3}(\dot{x}(t-$ $d), t)$ are unknown nonlinear perturbations. They satisfy that $f_{1}(0, t)=0, f_{2}(0, t)=0, f_{3}(0, t)=0$, and

$$
\begin{gathered}
f_{1}^{T}(x(t), t) f_{1}(x(t), t) \leq \alpha^{2} x^{T}(t) x(t), \\
f_{2}^{T}(x(t-h(t)), t) f_{2}(x(t-h(t)), t) \\
\leq \beta^{2} x^{T}(t-h(t)) x(t-h(t)), \\
f_{3}^{T}(\dot{x}(t-d), t) f_{3}(\dot{x}(t-d), t) \\
\leq \gamma^{2} \dot{x}^{T}(t-d) \dot{x}(t-d),
\end{gathered}
$$

where $\alpha \geq 0, \beta \geq 0$, and $\gamma \geq 0$ are given constants and, for simplicity, $f_{1}:=f_{1}(x(t), t), f_{2}:=f_{2}(x(t-h(t)), t)$, and $f_{3}=f_{3}(\dot{x}(t-d), t)$.

In this paper, we define the following scalar with respect to the variation range of time delay which is

$$
\bar{h}=\delta h \quad(0<\delta<1) .
$$

It is easy to see that for all $t \in \mathbf{R}^{+}$, we have $h(t) \in[0, \bar{h}]$ or $h(t) \in(\bar{h}, h]$. Consequently, in the proof of our main results, we will derive the delay-dependent stability criterion for two sets, respectively.

Before proceeding further, we will state the following well-known lemmas and definition.

Lemma 1 (see [12]). For any positive semidefinite matrices,

$$
X=\left(\begin{array}{ccc}
X_{11} & X_{12} & X_{13} \\
X_{12}^{T} & X_{22} & X_{23} \\
X_{13}^{T} & X_{23}^{T} & X_{23}
\end{array}\right) \geq 0,
$$

the following integral inequality holds:

$$
\begin{aligned}
&-\int_{t-h(t)}^{t} \dot{x}^{T}(s) X_{33} \dot{x}(s) d s \\
& \leq \int_{t-h(t)}^{t}\left(x^{T}(t), x^{T}(t-h(t)), \dot{x}^{T}(s)\right) \\
& \\
& \times\left(\begin{array}{ccc}
X_{11} & X_{12} & X_{13} \\
X_{12}^{T} & X_{22} & X_{23} \\
X_{13}^{T} & X_{23}^{T} & 0
\end{array}\right)\left(\begin{array}{c}
x(t) \\
x(t-h(t)) \\
\dot{x}(s)
\end{array}\right) d s .
\end{aligned}
$$

Lemma 2 (Schur complement). Given one positive definite matrix $S_{2}>0$ and constant matrices $S_{1}, S_{3}$, where $S_{1}=S_{1}^{T}$, then $S_{1}+S_{3}^{T} S_{2}^{-1} S_{3}<0$ if and only if

$$
\left(\begin{array}{cc}
S_{1} & S_{3}^{T} \\
S_{3} & -S_{2}
\end{array}\right)<0 \quad \text { or }\left(\begin{array}{cc}
-S_{2} & S_{3} \\
S_{3}^{T} & S_{1}
\end{array}\right)<0 .
$$

The operator $\mathscr{D}: \mathbf{C}\left([-d, 0], \mathbf{R}^{n}\right) \rightarrow \mathbf{R}^{n}$ is defined to be $\mathscr{D}(t)=x(t)-C x(t-d)$. Its stability is defined as follows.

Definition 3 (see [21]). The operator $\mathscr{D}$ is said to be stable if the zero solution of the homogeneous difference equation $\mathscr{D}(t)=0, t \geq 0, x_{0}=\psi \in\{\phi \in \mathbf{C}([-d, 0]: \mathscr{D} \phi=0)\}$ is uniformly asymptotically stable.

\section{Stability Analysis}

In this section, we first present a delay-dependent robust criterion for the system (1) with uncertainty (3), with a delay decomposition approach.

Theorem 4. If $0<h(t) \leq \bar{h}$, for given scalars $\alpha$, $\beta$, and $\gamma$, system (1) with uncertainty (3) is robustly stable if $\mathscr{D}$ is stable and there exist positive semidefinite matrices,

$$
X=\left(\begin{array}{ccc}
X_{11} & X_{12} & X_{13} \\
X_{12}^{T} & X_{22} & X_{23} \\
X_{13}^{T} & X_{23}^{T} & X_{23}
\end{array}\right) \geq 0,
$$




$$
\begin{aligned}
& Y=\left(\begin{array}{ccc}
Y_{11} & Y_{12} & Y_{13} \\
Y_{12}^{T} & Y_{22} & Y_{23} \\
Y_{13}^{T} & Y_{23}^{T} & Y_{23}
\end{array}\right) \geq 0 \\
& Z=\left(\begin{array}{ccc}
Z_{11} & Z_{12} & Z_{13} \\
Z_{12}^{T} & Z_{22} & Z_{23} \\
Z_{13}^{T} & Z_{23}^{T} & Z_{23}
\end{array}\right) \geq 0, \\
& \Theta=\left(\begin{array}{cc}
\Theta_{1} & \Gamma^{T} \Lambda \\
\Lambda^{T} \Gamma & -\Lambda
\end{array}\right)<0, \\
& R_{1}-X_{33} \geq 0, \quad R_{2}-Y_{33} \geq 0, \quad R_{1}+R_{3}-Z_{33} \geq 0
\end{aligned}
$$

(8) where

$$
\begin{aligned}
& \Gamma=(A, B, 0,0,0, C, I, I, I), \\
& \Lambda=W+\delta h R_{1}+(1-\delta) h R_{2}+h R_{3} \text {, } \\
& \Theta_{1}=\left(\begin{array}{ccccccccc}
\Theta_{11} & \Theta_{12} & -A^{T} P C & 0 & 0 & 0 & P & P & P \\
* & \Theta_{22} & -B^{T} P C & 0 & \Theta_{25} & 0 & 0 & 0 & 0 \\
* & * & -R & 0 & 0 & 0 & -C^{T} P & -C^{T} P & -C^{T} P \\
* & * & * & -W+\varepsilon_{3} \gamma^{2} I & 0 & 0 & 0 & 0 & 0 \\
* & * & * & * & \Theta_{55} & \Theta_{56} & 0 & 0 & 0 \\
* & * & * & * & * & \Theta_{66} & 0 & 0 & 0 \\
* & * & * & * & * & * & -\varepsilon_{1} I & 0 & 0 \\
* & * & * & * & * & * & * & -\varepsilon_{2} I & 0 \\
* & * & * & * & * & * & * & * & -\varepsilon_{3} I
\end{array}\right),
\end{aligned}
$$

with

$$
\begin{aligned}
\Theta_{11}= & P A+A^{T} P+R+Q_{1}+Q_{3}+\delta h Z_{11} \\
& +Z_{13}+Z_{13}^{T}+\varepsilon_{1} \alpha^{2} I, \\
\Theta_{12}= & P B+\delta h Z_{12}-Z_{13}+Z_{23}^{T}, \\
\Theta_{22}= & -\left(1-h_{d}\right) Q_{3}+\delta h X_{11}+X_{13}+X_{13}^{T} \\
& +\delta h Z_{22}-Z_{23}-Z_{23}^{T}+\varepsilon_{2} \beta^{2} I, \\
\Theta_{25}= & \delta h X_{12}-X_{13}+X_{23}^{T}, \\
\Theta_{55}= & Q_{2}-Q_{1}+\delta h X_{22}-X_{23}-X_{23}^{T} \\
& +(1-\delta) h Y_{11}+Y_{13}+Y_{13}^{T}, \\
\Theta_{56}= & (1-\delta) h Y_{12}-Y_{13}+Y_{23}^{T}, \\
\Theta_{66}= & -Q_{2}+(1-\delta) h Y_{22}-Y_{23}-Y_{23}^{T} .
\end{aligned}
$$

Proof. Choose a Lyapunov functional candidate for the system (1) to be

$$
V(t)=V_{1}(t)+V_{2}(t)+V_{3}(t)+V_{4}(t)+V_{5}(t),
$$

where

$$
\begin{aligned}
V_{1}(t)= & \mathscr{D}^{T}\left(x_{t}\right) P \mathscr{D}\left(x_{t}\right), \\
V_{2}(t)= & \int_{t-d}^{t} x^{T}(s) R x(s) d s+\int_{t-d}^{t} \dot{x}^{T}(s) W \dot{x}(s) d s, \\
V_{3}(t)= & \int_{t-\delta h}^{t} x^{T}(s) Q_{1} x(s) d s+\int_{t-h}^{t-\delta h} x^{T}(s) Q_{2} x(s) d s \\
& +\int_{t-h(t)}^{t} x^{T}(s) Q_{3} x(s) d s, \\
V_{4}(t)= & \int_{-\delta h}^{0} \int_{t+\theta}^{t} d \dot{x}^{T}(s) R_{1} \dot{x}(s) d s d \theta \\
& +\int_{-h}^{-\delta h} \int_{t+\theta}^{t} d \dot{x}^{T}(s) R_{2} \dot{x}(s) d s d \theta \\
& +\int_{-h}^{0} \int_{t+\theta}^{t} d \dot{x}^{T}(s) R_{3} \dot{x}(s) d s d \theta,
\end{aligned}
$$

where $P=P^{T}>0, R=R^{T}>0, W=W^{T}>0, R_{i}=R_{i}^{T}>0$, and $Q_{i}=Q_{i}^{T}>0(i=1,2,3)$ are to be determined. 
Next, from (3), we can obtain for any scalars $\varepsilon_{1}>0, \varepsilon_{2}>0$, and $\varepsilon_{3}>0$,

$$
\begin{gathered}
\varepsilon_{1}\left(\alpha^{2} x^{T}(t) x(t)-f_{1}^{T}(x(t), t) f_{1}(x(t), t)\right) \geq 0, \\
\varepsilon_{2}\left(\beta^{2} x^{T}(t-h(t)) x(t-h(t))\right. \\
\left.\quad-f_{2}^{T}(x(t-h(t)), t) f_{2}(x(t-h(t)), t)\right) \geq 0, \\
\varepsilon_{3}\left(\gamma^{2} \dot{x}^{T}(t-d) \dot{x}(t-d)\right. \\
\left.\quad-f_{3}^{T}(\dot{x}(t-d), t) f_{3}(\dot{x}(t-d), t)\right) \geq 0 .
\end{gathered}
$$

Now calculate the derivative of $V(t)$ along the trajectory of the system (1); we derive

$$
\begin{aligned}
& \dot{V}_{1}(t)=2 \mathscr{D}^{T}\left(x_{t}\right) P \dot{D}\left(x_{t}\right) \\
& =2\left(x^{T}(t)-x^{T}(t-d) C^{T}\right) \\
& \times P\left(A x(t)+B x(t-h(t))+f_{1}+f_{2}+f_{3}\right) \\
& =x^{T}(t)\left(P A+A^{T} P\right) x(t)+2 x^{T}(t) P B x(t-h(t)) \\
& +2 x^{T}(t) P f_{1}+2 x^{T}(t) P f_{2} \\
& +2 x^{T}(t) P f_{3}-2 x^{T}(t-d) C^{T} P A x(t) \\
& -2 x^{T}(t-d) C^{T} P B x(t-h(t)) \\
& -2 x^{T}(t-d) C^{T} P f_{1}-2 x^{T}(t-d) C^{T} P f_{2} \\
& -2 x^{T}(t-d) C^{T} P f_{3}, \\
& \dot{V}_{2}(t)=x^{T}(t) R x(t)-x^{T}(t-d) R x(t-d) \\
& +\dot{x}^{T}(t) W \dot{x}(t)-\dot{x}^{T}(t-d) W \dot{x}(t-d), \\
& \dot{V}_{3}(t) \leq x^{T}(t)\left(Q_{1}+Q_{3}\right) x(t) \\
& +x^{T}(t-\delta h)\left(Q_{2}-Q_{1}\right) x(t-\delta h) \\
& -x^{T}(t-h) Q_{2} x(t-h) \\
& -\left(1-h_{d}\right) x^{T}(t-h(t)) Q_{3} x(t-h(t)), \\
& \dot{V}_{4}(t)=\dot{x}^{T}(t)\left(\delta h R_{1}+(1-\delta) h R_{2}+h R_{3}\right) \dot{x}(t) \\
& -\int_{t-\delta h}^{t} \dot{x}^{T}(s) R_{1} \dot{x}(s) d s-\int_{t-h}^{t-\delta h} \dot{x}^{T}(s) R_{1} \dot{x}(s) d s \\
& -\int_{t-h}^{t} \dot{x}^{T}(s) R_{1} \dot{x}(s) d s \\
& \leq \dot{x}^{T}(t)\left(\delta h R_{1}+(1-\delta) h R_{2}+h R_{3}\right) \dot{x}(t) \\
& -\int_{t-\delta h}^{t} \dot{x}^{T}(s) R_{1} \dot{x}(s) d s-\int_{t-h}^{t-\delta h} \dot{x}^{T}(s) R_{2} \dot{x}(s) d s \\
& -\int_{t-h(t)}^{t} \dot{x}^{T}(s) R_{3} \dot{x}(s) d s .
\end{aligned}
$$

Now, we estimate the upper bound of the last three terms in inequality (19) as

$$
\begin{aligned}
-\int_{t-\delta h}^{t} & \dot{x}^{T}(s) R_{1} \dot{x}(s) d s-\int_{t-h}^{t-\delta h} \dot{x}^{T}(s) R_{2} \dot{x}(s) d s \\
& -\int_{t-h(t)}^{t} \dot{x}^{T}(s) R_{3} \dot{x}(s) d s \\
= & -\int_{t-\delta h}^{t-h(t)} \dot{x}^{T}(s) R_{1} \dot{x}(s) d s-\int_{t-h}^{t-\delta h} \dot{x}^{T}(s) R_{2} \dot{x}(s) d s \\
& -\int_{t-h(t)}^{t} \dot{x}^{T}(s)\left(R_{1}+R_{3}\right) \dot{x}(s) d s \\
= & -\int_{t-\delta h}^{t-h(t)} \dot{x}^{T}(s)\left(R_{1}-X_{33}\right) \dot{x}(s) d s \\
& -\int_{t-h}^{t-\delta h} \dot{x}^{T}(s)\left(R_{2}-Y_{33}\right) \dot{x}(s) d s \\
& -\int_{t-h}^{t-\delta h} \dot{x}^{T}(s) Y_{33} \dot{x}(s) d s-\int_{t-h(t)}^{t} \dot{x}^{T}(s) Z_{33} \dot{x}(s) d s . \\
& -\int_{t-h(t)}^{t-h(t)} \dot{x}^{T}(s)\left(R_{1}+R_{3}-Z_{33}\right) \dot{x}(s) d s \\
& \dot{x}^{T}(s) X_{33} \dot{x}(s) d s \\
& \\
& \\
&
\end{aligned}
$$

By virtue of Lemma 1 , if $0<h(t) \leq \bar{h}$, we get

$$
\begin{aligned}
& -\int_{t-\delta h}^{t-h(t)} \dot{x}^{T}(s) X_{33} \dot{x}(s) d s \\
& \leq \int_{t-\delta h}^{t-h(t)}\left(x^{T}(t-h(t)), x^{T}(t-\delta h), \dot{x}^{T}(s)\right) \\
& \times\left(\begin{array}{ccc}
X_{11} & X_{12} & X_{13} \\
X_{12}^{T} & X_{22} & X_{23} \\
X_{13}^{T} & X_{23}^{T} & 0
\end{array}\right)\left(\begin{array}{c}
x(t-h(t)) \\
x(t-\delta h) \\
\dot{x}(s)
\end{array}\right) d s \\
& \leq x^{T}(t-h(t))\left(\delta h X_{11}+X_{13}^{T}+X_{13}\right) x(t-h(t)) \\
& +2 x^{T}(t-h(t))\left(\delta h X_{12}-X_{13}+X_{23}^{T}\right) x(t-\delta h) \\
& +x^{T}(t-\delta h)\left(\delta h X_{22}-X_{23}-X_{23}^{T}\right) x(t-\delta h) .
\end{aligned}
$$


Then, similarly, we obtain

$$
\begin{aligned}
&-\int_{t-h}^{t-\delta h} \dot{x}^{T}(s) Y_{33} \dot{x}(s) d s \\
& \leq x^{T}(t-\delta h)\left((1-\delta h) Y_{11}+Y_{13}^{T}+Y_{13}\right) x(t-\delta h) \\
&+2 x^{T}(t-\delta h)\left((1-\delta) h Y_{12}-Y_{13}+Y_{23}^{T}\right) x(t-h) \\
&+x^{T}(t-h)\left((1-\delta) h Y_{22}-Y_{23}-Y_{23}^{T}\right) x(t-h), \\
&-\int_{t-h(t)}^{t} \quad \dot{x}^{T}(s) Z_{33} \dot{x}(s) d s x^{T}(t)\left(\delta h Z_{11}+Z_{13}^{T}+Z_{13}\right) x(t) \\
& \quad+2 x^{T}(t)\left(\delta h Z_{12}-Z_{13}+Z_{23}^{T}\right) x(t-h(t)) \\
& \quad+x^{T}(t-h(t))\left(\delta h Z_{22}-Z_{23}-Z_{23}^{T}\right) x(t-h(t)) .
\end{aligned}
$$

Then combining (15)-(22) yields

$$
\begin{aligned}
\dot{V}(t) \leq & \dot{V}_{1}(t)+\dot{V}_{2}(t)+\dot{V}_{3}(t)+\dot{V}_{4}(t) \\
& +\varepsilon_{1}\left(\alpha^{2} x^{T}(t) x(t)-f_{1}^{T}(x(t), t) f_{1}(x(t), t)\right) \\
& +\varepsilon_{2}\left(\beta^{2} x^{T}(t-h(t)) x(t-h(t))\right. \\
& \left.\quad-f_{2}^{T}(x(t-h(t)), t) f_{2}(x(t-h(t)), t)\right) \\
& +\varepsilon_{3}\left(\gamma^{2} \dot{x}^{T}(t-d) \dot{x}(t-d)\right. \\
& \left.\quad-f_{3}^{T}(\dot{x}(t-d), t) f_{3}(\dot{x}(t-d), t)\right) \\
= & x^{T}(t)\left(P A+A^{T} P+R+Q_{1}+Q_{3}+\delta h Z_{11}\right. \\
& \left.+Z_{13}+Z_{13}^{T}+\varepsilon_{1} \alpha^{2} I\right) x(t) \\
& +2 x^{T}(t)\left(P B+\delta h Z_{12}-Z_{13}+Z_{23}^{T}\right) x(t-h(t)) \\
& +2 x^{T}(t)\left(-A^{T} P C\right) x(t-d)+2 x^{T}(t) P f_{1} \\
& +2 x^{T}(t-d)\left(-C^{T} P\right) f_{2}+2 x^{T}(t-d)\left(-C^{T} P\right) f_{3} \\
& +2 x^{T}(t) P f_{2}+2 x^{T}(t) P f_{3}+x^{T}(t-h(t)) \\
& \times\left(-\left(1-h_{d}\right) Q_{3}+\delta h X_{11}+X_{13}+X_{13}^{T}+\delta h Z_{22}\right. \\
& \left.-Z_{23}-Z_{23}^{T}+\varepsilon_{2} \beta^{2} I\right) x(t-h(t)) \\
+ & 2 x^{T}(t-h(t))\left(-B^{T} P C\right) x(t-d)
\end{aligned}
$$

$$
\begin{aligned}
& +x^{T}(t-\delta h)\left(Q_{2}-Q_{1}+\delta h X_{22}-X_{23}-X_{23}^{T}\right. \\
& \left.+(1-\delta) h Y_{11}+Y_{13}+Y_{13}^{T}\right) x(t-\delta h) \\
& +2 x^{T}(t-\delta h)\left((1-\delta) h Y_{12}-Y_{13}+Y_{23}^{T}\right) x(t-h) \\
& +\dot{x}^{T}(t)\left(W+\delta h R_{1}+(1-\delta) h R_{2}+\delta h R_{3}\right) \dot{x}(t) \\
& -\varepsilon_{1} f_{1}^{T} f_{1}-\varepsilon_{2} f_{2}^{T} f_{2}-\varepsilon_{3} f_{3}^{T} f_{3} \\
& \leq \xi^{T}(t)\left(\Theta_{1}+\Gamma^{T} \Lambda \Gamma\right) \xi(t) \\
& -\int_{t-\delta h}^{t-h(t)} \dot{x}^{T}(s)\left(R_{1}-X_{33}\right) \dot{x}(s) d s \\
& -\int_{t-h}^{t-\delta h} \dot{x}^{T}(s)\left(R_{2}-Y_{33}\right) \dot{x}(s) d s \\
& -\int_{t-h(t)}^{t} \dot{x}^{T}(s)\left(R_{1}+R_{3}-Z_{33}\right) \dot{x}(s) d s
\end{aligned}
$$

where

$$
\begin{gathered}
\xi(t)=\left[x^{T}(t), x^{T}(t-h(t)), x^{T}(t-d),\right. \\
\left.\dot{x}^{T}(t-d), f_{1}^{T}, f_{2}^{T}, f_{3}^{T}\right]^{T} .
\end{gathered}
$$

If $R_{1}-X_{33} \geq 0, R_{2}-Y_{33} \geq 0, R_{1}+R_{3}-Z_{33} \geq 0$, and $0 \leq h(t) \leq \delta h$, we can derive

$$
\Theta_{1}+\Gamma^{T} \Lambda \Gamma<0
$$

from (9) and the Schur complement. Obviously, from (25), we can get $\dot{V}(t)<0$. Therefore, according to [21], if there exist symmetric positive definite matrices $P>0, R>0, W>0$, $Q_{i}>0$, and $R_{i}>0(i=1,2,3)$ such that the LMIs (9) and (10) are satisfied, then system (1) is robustly stable. This completes the proof.

Theorem 5. If $\bar{h}<h(t) \leq h$, for given scalars $\alpha$, $\beta$, and $\gamma$, system (1) with uncertainty (3) is robustly stable if $\mathscr{D}$ is stable and there exist positive semidefinite matrices,

$$
X=\left(\begin{array}{ccc}
X_{11} & X_{12} & X_{13} \\
X_{12}^{T} & X_{22} & X_{23} \\
X_{13}^{T} & X_{23}^{T} & X_{23}
\end{array}\right) \geq 0
$$




$$
\begin{aligned}
& Y=\left(\begin{array}{lll}
Y_{11} & Y_{12} & Y_{13} \\
Y_{12}^{T} & Y_{22} & Y_{23} \\
Y_{13}^{T} & Y_{23}^{T} & Y_{23}
\end{array}\right) \geq 0 \\
& Z=\left(\begin{array}{ccc}
Z_{11} & Z_{12} & Z_{13} \\
Z_{12}^{T} & Z_{22} & Z_{23} \\
Z_{13}^{T} & Z_{23}^{T} & Z_{23}
\end{array}\right) \geq 0
\end{aligned}
$$

and positive definite matrices $P>0, R>0, W>0, Q_{i}>0$, and $R_{i}>0(i=1,2,3)$ such that the following symmetric linear matrix inequalities hold:

$$
\begin{gathered}
\widehat{\Theta}=\left(\begin{array}{cc}
\widehat{\Theta}_{1} & \Gamma^{T} \Lambda \\
\Lambda^{T} \Gamma & -\Lambda
\end{array}\right)<0, \\
R_{1}+R_{3}-X_{33} \geq 0, \\
R_{2}+R_{3}-Y_{33} \geq 0, \\
R_{2}-Z_{33} \geq 0,
\end{gathered}
$$

where

$$
\begin{aligned}
& \Gamma=(A, B, 0,0,0, C, I, I, I), \\
& \Lambda=W+\delta h R_{1}+(1-\delta) h R_{2}+h R_{3}, \\
& \widehat{\Theta}=\left(\begin{array}{ccccccccc}
\widehat{\Theta}_{11} & P B & -A^{T} P C & 0 & \widehat{\Theta}_{15} & 0 & P & P & P \\
* & \widehat{\Theta}_{22} & -B^{T} P C & 0 & \widehat{\Theta}_{25} & \widehat{\Theta}_{26} & 0 & 0 & 0 \\
* & * & -R & 0 & 0 & 0 & -C^{T} P & -C^{T} P & -C^{T} P \\
* & * & * & -W+\varepsilon_{3} \gamma^{2} I & 0 & 0 & 0 & 0 & 0 \\
* & * & * & * & \widehat{\Theta}_{55} & 0 & 0 & 0 & 0 \\
* & * & * & * & * & \widehat{\Theta}_{66} & 0 & 0 & 0 \\
* & * & * & * & * & * & -\varepsilon_{1} I & 0 & 0 \\
* & * & * & * & * & * & * & -\varepsilon_{2} I & 0 \\
* & * & * & * & * & * & * & * & -\varepsilon_{3} I
\end{array}\right),
\end{aligned}
$$

with

$$
\begin{aligned}
\widehat{\Theta}_{11}= & P A+A^{T} P+R+Q_{1}+Q_{3}+\delta h X_{11} \\
& +X_{13}+X_{13}^{T}+\varepsilon_{1} \alpha^{2} I, \\
\widehat{\Theta}_{15}= & \delta X_{12}-X_{13}+X_{23}^{T}, \\
\widehat{\Theta}_{22}= & -\left(1-h_{d}\right) Q_{3}+(1-\delta) h Y_{22}-Y_{23} \\
& -Y_{23}^{T}+(1-\delta) h Z_{11}+Z_{13}+Z_{13}^{T}+\varepsilon_{2} \beta^{2} I, \\
\widehat{\Theta}_{25}= & (1-\delta) h Y_{12}^{T}-Y_{13}^{T}+Y_{23}, \\
\widehat{\Theta}_{26}= & (1-\delta) h Z_{12}-Z_{13}+Y_{23}^{T}, \\
\widehat{\Theta}_{55}= & Q_{2}-Q_{1}+\delta h X_{22}-X_{23}-X_{23}^{T} \\
& +(1-\delta) h Y_{11}+Y_{13}+Y_{13}^{T}, \\
\widehat{\Theta}_{66}= & -Q_{2}+(1-\delta) h Z_{22}-Z_{23}-Z_{23}^{T} .
\end{aligned}
$$

Proof. If $\bar{h}<h(t) \leq h$, we get

$$
\begin{aligned}
-\int_{t-\delta h}^{t} \dot{x}^{T}(s) R_{1} \dot{x}(s) d s-\int_{t-h}^{t-\delta h} \dot{x}^{T}(s) R_{2} \dot{x}(s) d s \\
-\int_{t-h(t)}^{t} \dot{x}^{T}(s) R_{3} \dot{x}(s) d s \\
=-\int_{t-\delta h}^{t} \dot{x}^{T}(s)\left(R_{1}+R_{3}-X_{33}\right) \dot{x}(s) d s \\
-\int_{t-h(t)}^{t-\delta h} \dot{x}^{T}(s)\left(R_{2}+R_{3}-Y_{33}\right) \dot{x}(s) d s \\
-\int_{t-h}^{t-h(t)} \dot{x}^{T}(s)\left(R_{2}-Z_{33}\right) \dot{x}(s) d s \\
-\int_{t-\delta h}^{t} \dot{x}^{T}(s) X_{33} \dot{x}(s) d s \\
-\int_{t-h(t)}^{t-\delta h} \dot{x}^{T}(s) Y_{33} \dot{x}(s) d s \\
-\int_{t-h}^{t-h(t)} \dot{x}^{T}(s) Z_{33} \dot{x}(s) d s .
\end{aligned}
$$


By virtue of Lemma 1 , notice that $R_{1}+R_{3}-X_{33} \geq 0, R_{2}+$ $R_{3}-Y_{33} \geq 0$, and $R_{2}-Z_{33} \geq 0$; it yields

$$
\begin{aligned}
-\int_{t-\delta h}^{t} & \dot{x}^{T}(s) X_{33} \dot{x}(s) d s \\
\leq & x^{T}(t)\left(\delta h X_{11}+X_{13}^{T}+X_{13}\right) x(t) \\
& +2 x^{T}(t)\left(\delta h X_{12}-X_{13}+X_{23}^{T}\right) x(t-\delta h) \\
& +x^{T}(t-\delta h)\left(\delta h X_{22}-X_{23}-X_{23}^{T}\right) x(t-\delta h) \\
-\int_{t-h(t)}^{t-\delta h} & \dot{x}^{T}(s) Y_{33} \dot{x}(s) d s \\
\leq & x^{T}(t-\delta h)\left((1-\delta h) Y_{11}+Y_{13}^{T}+Y_{13}\right) x(t-\delta h) \\
& +2 x^{T}(t-\delta h)\left((1-\delta) h Y_{12}-Y_{13}+Y_{23}^{T}\right) x(t-h(t)) \\
& +x^{T}(t-h(t))\left((1-\delta) h Y_{22}-Y_{23}-Y_{23}^{T}\right) x(t-h(t))
\end{aligned}
$$

$$
\begin{aligned}
-\int_{t-h}^{t-h(t)} & \dot{x}^{T}(s) Z_{33} \dot{x}(s) d s \\
\leq & x^{T}(t-h(t))\left((1-\delta) h Z_{11}+Z_{13}^{T}+Z_{13}\right) x(t-h(t)) \\
& +2 x^{T}(t-h(t))\left((1-\delta) h Z_{12}-Z_{13}+Z_{23}^{T}\right) x(t-h) \\
& +x^{T}(t-h(t))\left((1-\delta) h Z_{22}-Z_{23}-Z_{23}^{T}\right) x(t-h) .
\end{aligned}
$$

Then combining (15)-(19) and (31)-(33) yields

$$
\begin{aligned}
\dot{V}(t) \leq & \xi^{T}(t)\left(\widehat{\Theta}_{1}+\Gamma^{T} \Lambda \Gamma\right) \xi(t) \\
& -\int_{t-\delta h}^{t} \dot{x}^{T}(s)\left(R_{1}+R_{3}-X_{33}\right) \dot{x}(s) d s \\
& -\int_{t-h(t)}^{t-\delta h} \dot{x}^{T}(s)\left(R_{2}+R_{3}-Y_{33}\right) \dot{x}(s) d s \\
& -\int_{t-h}^{t-h(t)} \dot{x}^{T}(s)\left(R_{2}-Z_{33}\right) \dot{x}(s) d s .
\end{aligned}
$$

Next, similar to Theorem 4, we can get the result of Theorem 5. This completes the proof.

Remark 6. Theorems 4 and 5 give the delay-dependent and rate-dependent robust stability criteria for system (1) with uncertainty (3) by employing delay decomposition approach as in [12]. The proposed criteria use as few as possible decision variables while showing less conservativeness in comparison to those in [7-11].
Remark 7. When $C=0$ and $f_{3}(\dot{x}(t-d), t) \equiv 0$, the system (1) can be reduced to the following system:

$$
\begin{gathered}
\dot{x}(t)=A x(t)+B x(t-h(t)) \\
+f_{1}(x(t), t)+f_{2}(x(t-h(t)), t), \\
x(\theta)=\phi(\theta), \quad \dot{x}(\theta)=\varphi(\theta), \quad \forall \theta \in[-h, 0] .
\end{gathered}
$$

We will show the obtained stability criteria for this case in Corollaries 8 and 9 .

Corollary 8. If $0<h(t) \leq \bar{h}$, for given scalars $\alpha$ and $\beta$, system (35) with uncertainty (3) is robustly stable if there exist positive semidefinite matrices,

$$
\begin{aligned}
& X=\left(\begin{array}{lll}
X_{11} & X_{12} & X_{13} \\
X_{12}^{T} & X_{22} & X_{23} \\
X_{13}^{T} & X_{23}^{T} & X_{23}
\end{array}\right) \geq 0 \\
& Y=\left(\begin{array}{lll}
Y_{11} & Y_{12} & Y_{13} \\
Y_{12}^{T} & Y_{22} & Y_{23} \\
Y_{13}^{T} & Y_{23}^{T} & Y_{23}
\end{array}\right) \geq 0 \\
& Z=\left(\begin{array}{lll}
Z_{11} & Z_{12} & Z_{13} \\
Z_{12}^{T} & Z_{22} & Z_{23} \\
Z_{13}^{T} & Z_{23}^{T} & Z_{23}
\end{array}\right) \geq 0
\end{aligned}
$$

and positive definite matrices $P>0, R>0, W>0, Q_{i}>0$, and $R_{i}>0(i=1,2,3)$ such that the following symmetric linear matrix inequalities hold:

$$
\begin{gathered}
\Pi=\left(\begin{array}{cc}
\Pi_{1} & \Gamma^{T} \widehat{\Lambda} \\
\widehat{\Lambda}^{T} \widehat{\Gamma} & -\widehat{\Lambda}
\end{array}\right)<0, \\
R_{1}-X_{33} \geq 0, \quad R_{2}-Y_{33} \geq 0,
\end{gathered}
$$

where

$$
\begin{gathered}
\widehat{\Gamma}=(A, B, 0,0, I, I, I), \\
\widehat{\Lambda}=\delta h R_{1}+(1-\delta) h R_{2}+h R_{3}, \\
\Pi_{1}=\left(\begin{array}{ccccccc}
\Pi_{11} & \Pi_{12} & 0 & 0 & P & P & P \\
* & \Pi_{22} & \Pi_{23} & 0 & 0 & 0 & 0 \\
* & * & \Pi_{33} & \Pi_{34} & 0 & 0 & 0 \\
* & * & * & \Pi_{44} & 0 & 0 & 0 \\
* & * & * & * & -\varepsilon_{1} I & 0 & 0 \\
* & * & * & * & * & -\varepsilon_{2} I & 0 \\
* & * & * & * & * & * & -\varepsilon_{3} I
\end{array}\right),
\end{gathered}
$$


with

$$
\begin{aligned}
\Pi_{11}= & P A+A^{T} P+Q_{1}+Q_{3}+\delta h Z_{11}+Z_{13}+Z_{13}^{T}+\varepsilon_{1} \alpha^{2} I \\
\Pi_{12}= & P B+\delta h Z_{12}-Z_{13}+Z_{23}^{T}, \\
\Pi_{22}= & \left(1-h_{d}\right) Q_{3}+\delta h X_{11}+X_{13}+X_{13}^{T}+\delta h Z_{22} \\
& -Z_{23}-Z_{23}^{T}+\varepsilon_{2} \beta^{2} I \\
\Pi_{23}= & \delta h X_{12}-X_{13}+X_{23}^{T}, \\
\Pi_{33}= & Q_{2}-Q_{1}+\delta h X_{22}-X_{23}-X_{23}^{T} \\
& +(1-\delta) h Y_{11}+Y_{13}+Y_{13}^{T}, \\
\Pi_{34}= & (1-\delta) h Y_{12}-Y_{13}+Y_{23}^{T}, \\
\Pi_{44}= & -Q_{2}+(1-\delta) h Y_{22}-Y_{23}-Y_{23}^{T} .
\end{aligned}
$$

Corollary 9. If $\bar{h}<h(t) \leq h$, for given scalars $\alpha$ and $\beta$, system (35) with uncertainty (3) is robustly stable if there exist positive semidefinite matrices

$$
\begin{aligned}
& X=\left(\begin{array}{lll}
X_{11} & X_{12} & X_{13} \\
X_{12}^{T} & X_{22} & X_{23} \\
X_{13}^{T} & X_{23}^{T} & X_{23}
\end{array}\right) \geq 0 \\
& Y=\left(\begin{array}{ccc}
Y_{11} & Y_{12} & Y_{13} \\
Y_{12}^{T} & Y_{22} & Y_{23} \\
Y_{13}^{T} & Y_{23}^{T} & Y_{23}
\end{array}\right) \geq 0 \\
& Z=\left(\begin{array}{lll}
Z_{11} & Z_{12} & Z_{13} \\
Z_{12}^{T} & Z_{22} & Z_{23} \\
Z_{13}^{T} & Z_{23}^{T} & Z_{23}
\end{array}\right) \geq 0
\end{aligned}
$$

and positive definite matrices $P>0, R>0, W>0, Q_{i}>0$, and $R_{i}>0(i=1,2,3)$ such that the following symmetric linear matrix inequalities hold:

$$
\begin{gathered}
\widehat{\Pi}=\left(\begin{array}{cc}
\widehat{\Pi}_{1} & \widehat{\Gamma}^{T} \widehat{\Lambda} \\
\widehat{\Lambda}^{T} \widehat{\Gamma} & -\widehat{\Lambda}
\end{array}\right)<0, \\
R_{1}+R_{3}-Z_{33} \geq 0, \quad R_{2}-Z_{33} \geq 0,
\end{gathered}
$$

where

$$
\begin{gathered}
\widehat{\Gamma}=(A, B, 0,0, I, I, I) \\
\widehat{\Pi}_{1}=\left(\begin{array}{ccccccc}
\widehat{\Pi}_{11} & P B & \widehat{\Pi}_{13} & 0 & P & P & P \\
* & \widehat{\Pi}_{22} & \widehat{\Pi}_{23} & 0 & 0 & 0 & 0 \\
* & * & \Pi_{33} & 0 & 0 & 0 & 0 \\
* & * & * & \Pi_{44} & 0 & 0 & 0 \\
* & * & * & * & -\varepsilon_{1} I & 0 & 0 \\
* & * & * & * & * & -\varepsilon_{2} I & 0 \\
* & * & * & * & * & * & -\varepsilon_{3} I
\end{array}\right),
\end{gathered}
$$

with

$$
\begin{aligned}
\widehat{\Pi}_{11}= & P A+A^{T} P+Q_{1}+Q_{3}+\delta h X_{11} \\
& +X_{13}+X_{13}^{T}+\varepsilon_{1} \alpha^{2} I, \\
\widehat{\Pi}_{13}= & \delta X_{12}-X_{13}+X_{23}^{T}, \\
\widehat{\Pi}_{22}= & -\left(1-h_{d}\right) Q_{3}+(1-\delta) h Y_{22}-Y_{23} \\
& -Y_{23}^{T}+(1-\delta) h Z_{11}+Z_{13}+Z_{13}^{T}+\varepsilon_{2} \beta^{2} I, \\
\widehat{\Pi}_{23}= & (1-\delta) h Y_{12}^{T}-Y_{13}^{T}+Y_{23}, \\
\widehat{\Pi}_{24}= & (1-\delta) h Z_{12}-Z_{13}+Y_{23}^{T}, \\
\widehat{\Pi}_{44}= & -Q_{2}+(1-\delta) h Z_{22}-Z_{23}-Z_{23}^{T} .
\end{aligned}
$$

Remark 10. The norm-bounded uncertainties can be treated as a special case of nonlinear perturbations. Then, the criteria obtained in Theorems 4 and 5 and Corollaries 8 and 9 can also be applicable to the system with norm-bounded uncertainties.

Remark 11. Note that the interval $[t-h, t]$ is divided into subintervals $[t-h, t-\delta h]$ and $[t-\delta h, t]$ in the proof of Theorems 4 and 5 and Corollaries 8 and 9 , and then the information of delayed state $x(t-\delta h)$ can be taken into account. It is clear that the Lyapunov functional defined in our results is more general than the ones in $[2,3,7-11,14,20]$ which can be seen from the following example.

\section{Illustrative Example}

In this section, an example is presented to illustrate the effectiveness of the stability method proposed in this paper.

Example 1. Consider the following system as in [1] with

$$
\begin{gathered}
A=\left(\begin{array}{cc}
-1.2 & 0.1 \\
-0.1 & -1
\end{array}\right), \\
B=\left(\begin{array}{cc}
-0.6 & 0.7 \\
-1 & -0.8
\end{array}\right), \\
C=\left(\begin{array}{ll}
c & 0 \\
0 & c
\end{array}\right),
\end{gathered}
$$


TABLE 1: Maximum upper bound of $h$ with $h_{d}=0.5$ and different values of $\gamma$.

\begin{tabular}{|c|c|c|c|c|c|c|c|c|}
\hline \multirow[t]{2}{*}{$\gamma$} & \multicolumn{4}{|c|}{$\alpha=0$} & \multicolumn{4}{|c|}{$\alpha=0.1$} \\
\hline & 0 & 0.1 & 0.2 & 0.3 & 0 & 0.1 & 0.2 & 0.3 \\
\hline Rakkiyappan et al. [9] & 1.4886 & 1.2437 & 0.9921 & 0.7367 & 1.3244 & 1.0901 & 0.8475 & 0.6300 \\
\hline Qiu et al. [8] & 1.5998 & 1.3998 & 1.1998 & 0.9998 & 1.5998 & 1.3998 & 1.1998 & 0.9998 \\
\hline Lakshmanan et al. [10] & 1.6325 & 1.3386 & 1.0816 & 0.8563 & 1.4440 & 1.1950 & 0.9734 & 0.7760 \\
\hline Cheng et al. [11] & 1.6865 & 1.3721 & 1.0923 & 0.8613 & 1.4721 & 1.2466 & 0.9996 & 0.7804 \\
\hline Theorem $4(\delta=0.4)$ & 2.2937 & 1.8505 & 1.4565 & 1.1105 & 2.0417 & 1.6541 & 1.3062 & 0.9982 \\
\hline
\end{tabular}

TABLE 2: Maximum upper bound of $d=h$ with different values of $\gamma$.

\begin{tabular}{lcccccc}
\hline$c$ & 0.1 & 0.2 & 0.3 & 0.4 & 0.5 & 0.6 \\
\hline Zhang and Yu [3] & 0.4911 & 0.4125 & 0.3382 & 0.2671 & 0.1975 & 0.1294 \\
Qiu et al. [7] & 1.8567 & 1.6242 & 1.3917 & 1.1592 & 0.9270 & 0.6945 \\
Theorem 4 & $2.1916(\delta=0.25)$ & $1.6632(\delta=0.25)$ & $1.4743(\delta=0.2)$ & $1.2396(\delta=0.15)$ & $0.9288(\delta=0.1)$ & $0.7446(\delta=0.02)$ \\
\hline
\end{tabular}

$$
\begin{gathered}
f_{1}^{T}(x(t), t) f_{1}(x(t), t) \leq \alpha^{2} x^{T}(t) x(t), \\
f_{2}^{T}(x(t-\tau(t)), t) f_{2}(x(t-\tau(t)), t) \\
\leq \beta^{2} x^{T}(t-\tau(t)) x(t-\tau(t)), \\
f_{3}^{T}(\dot{x}(t-d), t) f_{3}(\dot{x}(t-d), t) \\
\leq \gamma^{2} \dot{x}^{T}(t-d) \dot{x}(t-d),
\end{gathered}
$$

where $0 \leq|c|<1, \alpha \geq 0, \beta \geq 0$, and $\gamma \geq 0$.

Case I. For $c=0.1, \beta=0.1, d=1$, and $h_{d}=0.5$ and different values of $\gamma$, we consider the maximal allowable value $h$ that guarantees the robust stability of the system by applying criteria in [8-11] and in this work. Table 1 illustrates the numerical results for different $\gamma, \alpha=0$ and $\alpha=0.1$, respectively. From Table 1, one can see that the maximum allowable delay $h$ decreases as $\gamma$ increases. In addition, it is easy to see that our proposed stability criterion gives a much less conservative result than those in [8-11]. For example, when $\alpha=0.1, \gamma=0.1$, and $h_{d}=0.5$, by solving LMI (13) of Theorem 4, we obtain the maximum bound $h=1.6541$ with the following solutions:

$$
\begin{aligned}
P & =\left(\begin{array}{ll}
6.4750 & 0.3339 \\
0.3339 & 4.8054
\end{array}\right), \\
R & =\left(\begin{array}{ll}
1.2019 & 0.0620 \\
0.0620 & 0.8920
\end{array}\right), \\
Q_{1} & =\left(\begin{array}{ll}
1.6922 & 0.1468 \\
0.1468 & 1.2747
\end{array}\right), \\
Q_{2} & =\left(\begin{array}{ll}
0.7128 \times 10^{-4} & 0.0178 \times 10^{-4} \\
0.0178 \times 10^{-4} & 0.4708 \times 10^{-4}
\end{array}\right), \\
Q_{3} & =\left(\begin{array}{ll}
5.2627 & 0.5743 \\
0.5743 & 3.1830
\end{array}\right),
\end{aligned}
$$

$$
\begin{aligned}
& W=\left(\begin{array}{ll}
0.5738 & 0.0172 \\
0.0172 & 0.5248
\end{array}\right), \\
& R_{1}=\left(\begin{array}{ll}
2.7028 & 0.2344 \\
0.2344 & 2.0361
\end{array}\right), \\
& R_{2}=\left(\begin{array}{ll}
0.2278 \times 10^{-4} & 0.0059 \times 10^{-4} \\
0.0059 \times 10^{-4} & 0.1477 \times 10^{-4}
\end{array}\right), \\
& R_{3}=\left(\begin{array}{ll}
0.5931 \times 10^{-5} & 0.0145 \times 10^{-4} \\
0.0145 \times 10^{-4} & 0.3964 \times 10^{-4}
\end{array}\right) .
\end{aligned}
$$

Case II. For $\alpha=0.1, \beta=0.2, \gamma=0.1$, and $h_{d}=1$ and different values of $c$, the maximum upper bounds on the allowable delay of $h=d$ obtained from Theorem 4 are listed in Table 2. As $c$ increases, $h$ decreases. It can be seen from Table 2 that the proposed method in this work provides a considerably less conservative delay bound in comparison to the ones in $[3,7]$. For example, when $c=0.2$, by solving LMI (13) of Theorem 4 , we obtain the maximum bound $h=d=$ 1.6632 with the following solutions:

$$
\begin{aligned}
P & =\left(\begin{array}{ll}
3.6366 & 0.1165 \\
0.1165 & 2.6811
\end{array}\right), \\
R & =\left(\begin{array}{ll}
1.7371 & 0.0556 \\
0.0556 & 1.2806
\end{array}\right), \\
Q_{1} & =\left(\begin{array}{ll}
2.0254 & 0.1910 \\
0.1910 & 1.5301
\end{array}\right), \\
Q_{2} & =\left(\begin{array}{ll}
0.1438 \times 10^{-3} & 0.0030 \times 10^{-3} \\
0.0030 \times 10^{-3} & 0.1027 \times 10^{-3}
\end{array}\right), \\
Q_{3} & =\left(\begin{array}{ll}
0.4922 \times 10^{-4} & 0.0104 \times 10^{-4} \\
0.0104 \times 10^{-4} & 0.3515 \times 10^{-4}
\end{array}\right), \\
W & =\left(\begin{array}{ll}
0.3276 & 0.0152 \\
0.0152 & 0.2882
\end{array}\right),
\end{aligned}
$$


TABLE 3: Maximum upper bound of $h$ with $\beta=0.1$.

\begin{tabular}{|c|c|c|c|c|c|c|}
\hline & \multicolumn{3}{|c|}{$\alpha=0$} & \multicolumn{3}{|c|}{$\alpha=0.1$} \\
\hline & $h_{d}=0$ & $h_{d}=0.5$ & $h_{d} \geq 1$ & $h_{d}=0$ & $h_{d}=0.5$ & $h_{d} \geq 1$ \\
\hline Zou and Wang [14] & 2.7422 & 1.1424 & - & 1.8753 & 1.0097 & - \\
\hline Chen et al. [2] & 2.7423 & 1.1425 & 0.7355 & 1.8753 & 1.0097 & 0.7147 \\
\hline Qiu et al. [7] & 2.7757 & 1.1849 & 0.9284 & 1.8959 & 1.0512 & 0.8865 \\
\hline Zhang et al. [20] & - & 1.442 & 1.280 & - & 1.284 & 1.209 \\
\hline Corollary $8(\delta=0.72)$ & 3.8066 & 1.6402 & 1.2869 & 2.6039 & 1.4534 & 1.228 \\
\hline Corollary $9(\delta=0.79)$ & 2.8261 & 1.5213 & 1.5212 & 1.9233 & 1.3095 & 1.3095 \\
\hline
\end{tabular}

$$
\begin{aligned}
& R_{1}=\left(\begin{array}{ll}
1.5492 & 0.1461 \\
0.1461 & 1.1704
\end{array}\right), \\
& R_{2}=\left(\begin{array}{ll}
0.1373 \times 10^{-4} & 0.0032 \times 10^{-4} \\
0.0032 \times 10^{-4} & 0.0944 \times 10^{-4}
\end{array}\right), \\
& R_{3}=\left(\begin{array}{ll}
0.4269 \times 10^{-5} & 0.0088 \times 10^{-5} \\
0.0088 \times 10^{-5} & 0.3079 \times 10^{-5}
\end{array}\right) .
\end{aligned}
$$

Case III. For $C=0$ and $f_{3}(\dot{x}(t-d), t)=0$, the maximum value $h$ obtained from Corollaries 8 and 9 is listed in Table 3. It is clear that the obtained results in our paper are significantly better than those in $[2,7,14,20]$.

\section{Conclusion}

This paper has discussed robust stability problem for neutral systems with mixed delays and nonlinear perturbations. Based on the Lyapunov method and linear matrix inequality technology, delay-dependent stability conditions are derived by using a delay decomposition approach. The proposed criterion is both delay-dependent and rate-dependent. A numerical example has shown the less conservatism of the proposed method.

\section{Conflict of Interests}

The authors declare that there is no conflict of interests regarding the publication of this paper.

\section{Acknowledgments}

This work was funded by the Science Foundation of China Postdoctoral (no. 2013M531245), the Postdoctoral Research Funds of Jiangsu Planned Projects of China (no. 1302016C), the Natural Science Foundation of Shandong Province (no. ZR2013AM003), the Research Fund for the Doctors of Binzhou University (no. 2010Y09), and the Subject Construction Funds of Mathematics in Binzhou University.

\section{References}

[1] Q. L. Han and L. Yu, "Robust stability of linear neutral systems with nonlinear parameter perturbations," IEE Proceedings on Control Theory and Applications, vol. 15, no. 5, pp. 539-546, 2004.
[2] Y. Chen, A. Xue, R. Lu, and S. Zhou, "On robustly exponential stability of uncertain neutral systems with time-varying delays and nonlinear perturbations," Nonlinear Analysis: Theory, Methods \& Applications, vol. 68, no. 8, pp. 2464-2470, 2008.

[3] W. Zhang and L. Yu, "Delay-dependent robust stability of neutral systems with mixed delays and nonlinear perturbations," Acta Automatica Sinica, vol. 33, no. 8, pp. 863-866, 2007.

[4] Y. He, Q. Wang, C. Lin, and M. Wu, "Augmented Lyapunov functional and delay-dependent stability criteria for neutral systems," International Journal of Robust and Nonlinear Control, vol. 15, no. 18, pp. 923-933, 2005.

[5] X. M. Zhang and Q. L. Han, "Stability analysis and $H_{\infty}$ filtering for delay differential systems of neutral type," IET Control Theory \& Applications, vol. 1, no. 3, pp. 749-755, 2007.

[6] M. N. A. Parlakçı, "Robust stability of uncertain neutral systems: a novel augmented Lyapunov functional approach," IET Control Theory \& Applications, vol. 1, no. 3, pp. 802-809, 2007.

[7] F. Qiu, B. Cui, and Y. Ji, "Further results on robust stability of neutral system with mixed time-varying delays and nonlinear perturbations," Nonlinear Analysis: Real World Applications, vol. 11, no. 2, pp. 895-906, 2010.

[8] F. Qiu, B. T. Cui, and Y. Ji, "A delay-dividing approach to stability of neutral system with mixed delays and nonlinear perturbations," Applied Mathematical Modelling, vol. 34, no. 11, pp. 3701-3707, 2010.

[9] R. Rakkiyappan, P. Balasubramaniam, and R. Krishnasamy, "Delay dependent stability analysis of neutral systems with mixed time-varying delays and nonlinear perturbations," Journal of Computational and Applied Mathematics, vol. 235, no. 8, pp. 2147-2156, 2011.

[10] S. Lakshmanan, T. Senthilkumar, and P. Balasubramaniam, "Improved results on robust stability of neutral systems with mixed time-varying delays and nonlinear perturbations," Applied Mathematical Modelling. Simulation and Computation for Engineering and Environmental Systems, vol. 35, no. 11, pp. 5355-5368, 2011.

[11] J. Cheng, H. Zhu, S. M. Zhong, and G. H. Li, "Novel delay-dependent robust stability criteria for neutral systems with mixed time-varying delays and nonlinear perturbations," Applied Mathematics and Computation, vol. 219, no. 14, pp. 77417753, 2013.

[12] P. Liu, "A delay decomposition approach to stability analysis of neutral systems with time-varying delay," Applied Mathematical Modelling, vol. 37, no. 7, pp. 5013-5026, 2013.

[13] S. Xu and T. Chen, "Robust Ho control for uncertain stochastic systems with state delay," IEEE Transactions on Automatic Control, vol. 47, no. 12, pp. 2089-2094, 2002.

[14] Z. Zou and Y. Wang, "New stability criterion for a class of linear systems with time-varying delay and nonlinear perturbations," 
IEE Proceedings: Control Theory and Applications, vol. 153, no. 5, pp. 623-626, 2006.

[15] Y. S. Lee, Y. S. Moon, W. H. Kwon, and P. G. Park, "Delaydependent robust $\mathrm{H}_{\alpha}$ control for uncertain systems with a statedelay," Automatica, vol. 40, no. 1, pp. 65-72, 2004.

[16] Y. S. Moon, P. Park, and W. H. a. Kwon, "Delay-dependent robust stabilization of uncertain state-delayed systems," International Journal of Control, vol. 74, no. 14, pp. 1447-1455, 2001.

[17] Y. He, Q. G. Wang, L. H. Xie, and C. Lin, "Further improvement of free-weighting matrices technique for systems with timevarying delay," Transactions on Automatic Control, vol. 52, no. 2, pp. 293-299, 2007.

[18] Y. He, M. Wu, J.-H. She, and G.-P. Liu, "Parameter-dependent Lyapunov functional for stability of time-delay systems with polytopic-type uncertainties," IEEE Transactions on Automatic Control, vol. 49, no. 5, pp. 828-832, 2004.

[19] X. L. Zhu and G. H. Yang, "New results of stability analysis for systems with time-varying delay," International Journal of Robust and Nonlinear Control, vol. 20, no. 5, pp. 596-606, 2010.

[20] W. Zhang, X.-S. Cai, and Z.-Z. Han, "Robust stability criteria for systems with interval time-varying delay and nonlinear perturbations," Journal of Computational and Applied Mathematics, vol. 234, no. 1, pp. 174-180, 2010.

[21] J. K. Hale and S. M. Verduyn Lunel, Introduction to FunctionalDifferential Equations, vol. 99 of Applied Mathematical Sciences, Springer, New York, NY, USA, 1993. 


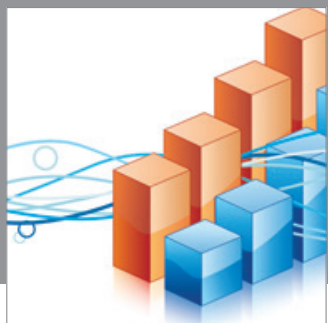

Advances in

Operations Research

mansans

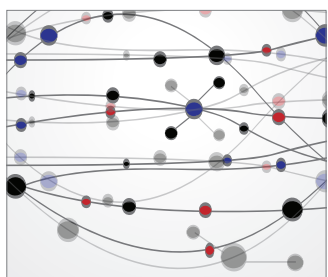

The Scientific World Journal
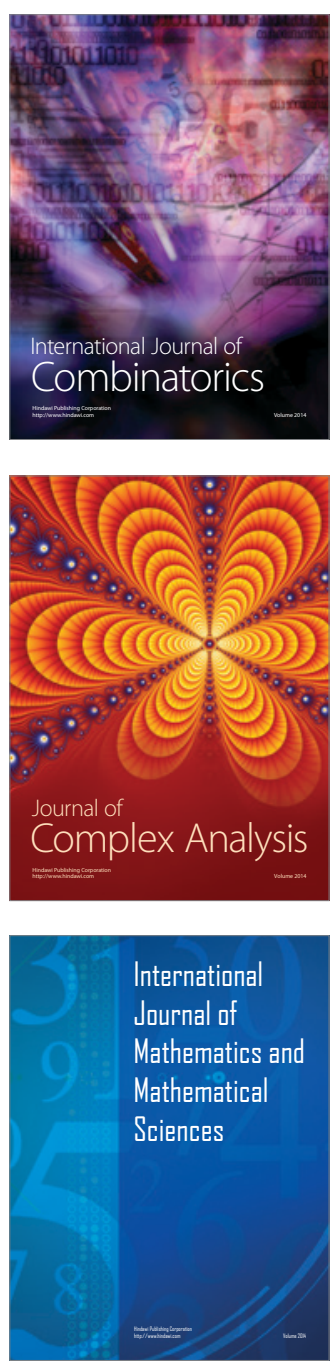
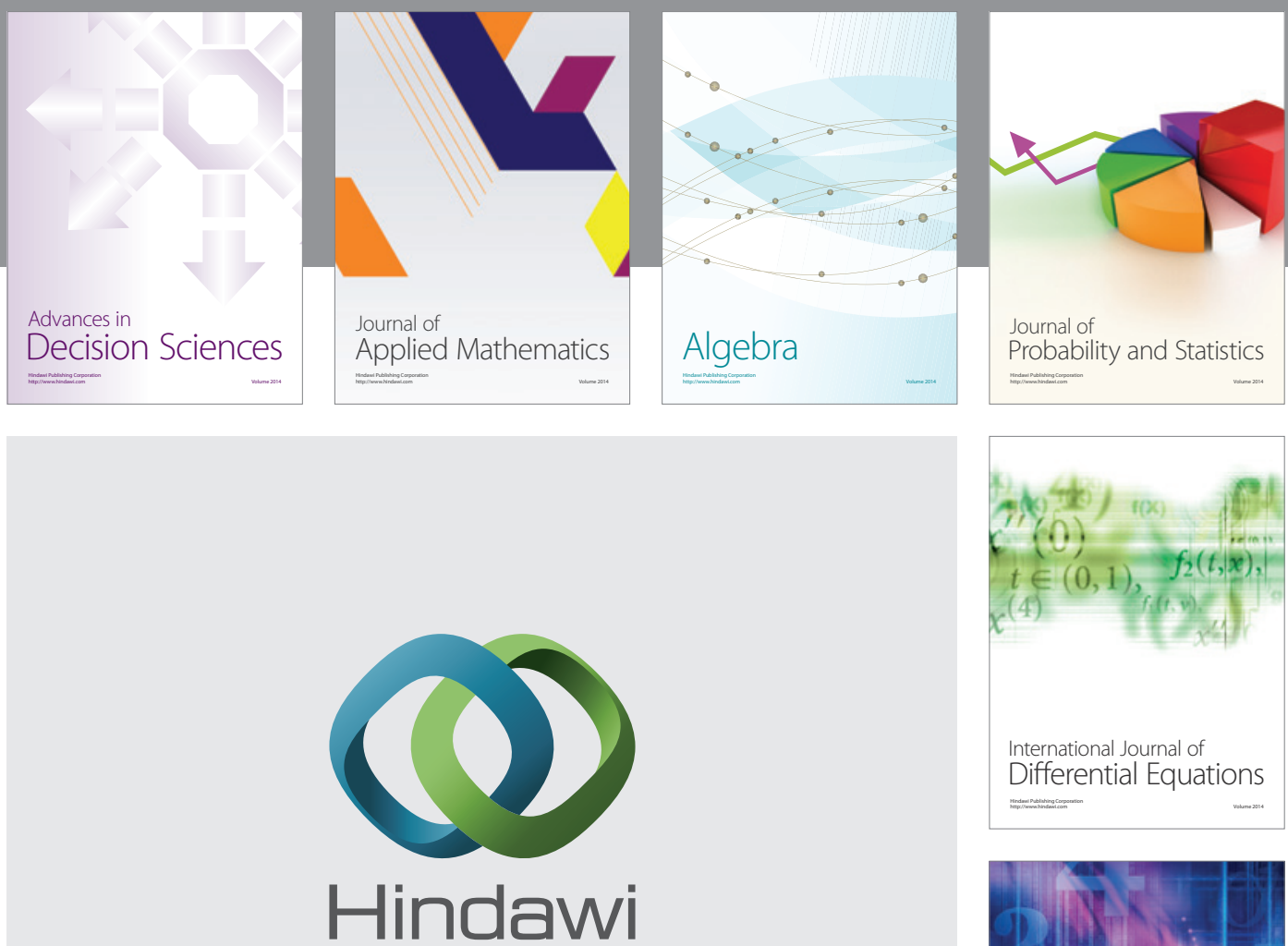

Submit your manuscripts at http://www.hindawi.com
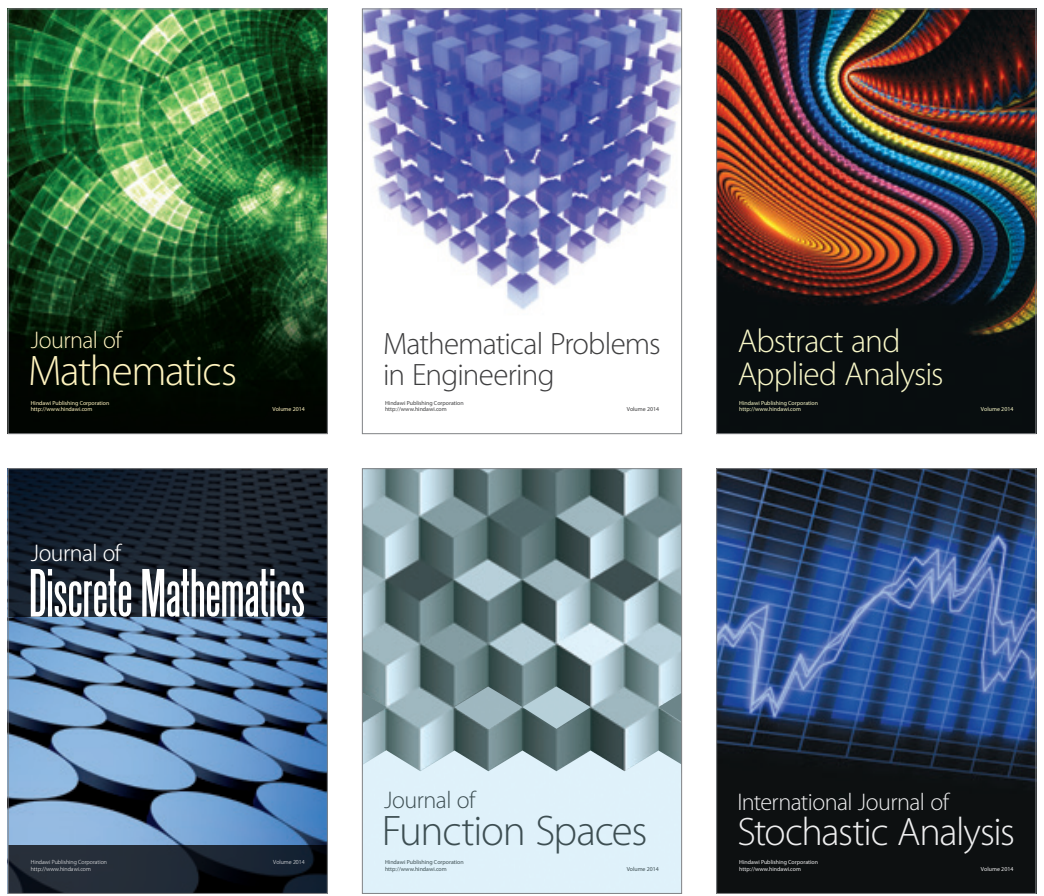

Journal of

Function Spaces

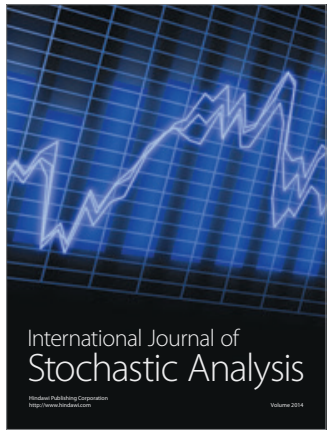

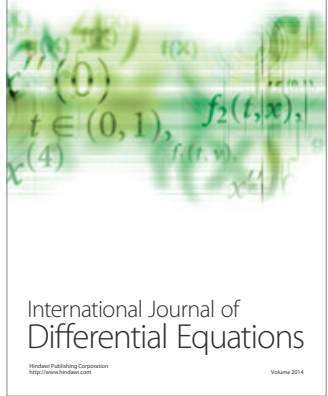
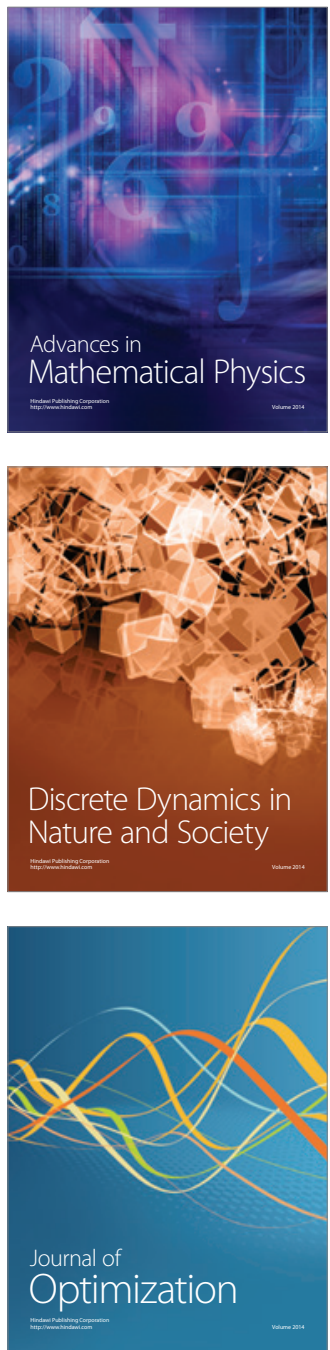